\title{
Prototipo para orientación de personas con discapacidad visual mediante una aplicación para móvil
}

\author{
Prototype for orientation of visually impaired people using a mobile application
}

\section{Protótipo para orientação de pessoas com deficiência visual utilizando uma aplicação móvel}

\author{
Dannyll Michellc Zambrano ${ }^{1}$ \\ Yohanna Daniela Daza-Álava² \\ José Daniel Pinargote-Zambrano ${ }^{3}$ \\ Edgar Danilo Lituma-Ramírez ${ }^{4}$
}

Recibido: febrero de 2019

Aceptado: abril de 2019

Para citar este artículo: Zambrano, D. M; Daza-Álava, Y. D; Pinargote-Zambrano, J.D; Lituma-Ramírez, E.D. (2019). Prototipo para orientación de personas con discapacidad visual mediante una aplicación para móvil. Revista Científica, 35(2), 247-257. Doi: https://doi.org/10.14483/23448350.14523

\section{Resumen}

El presente trabajo describe el diseño y elaboración de un prototipo que, por medio de un algoritmo, se vincule con una aplicación para móvil (App) y permita la orientación de personas con discapacidad visual puesto en marcha en la Facultad de Ciencias Informáticas de la Universidad Técnica de Manabí. Este se compone de dos partes, la aplicación móvil la cual se desarrolló en el entorno de programación Android Studio que permite detectar señales inalámbricas (estándar IEEE 802.11), por ello el dispositivo halla ciertas señales preprogramadas a cierta distancia y emite un audio que estará acompañado de una vibración distinta para cada punto de referencia indicando el sector por el cual se encuentra circulando. La otra parte del prototipo son los puntos de referencia o puntos de acceso, cuyo principal componente es un módulo inalámbrico (NodeMcu V3 LoLin); el cual mediante un algoritmo emite la señalización en la banda ISM de $2.4 \mathrm{GHZ}$ a los terminales móviles. Los puntos de referencia tienen una autonomía energética de la alimentación de corriente alterna de hasta 20 horas por medio de un panel fotovoltaico en un circuito de conmutación automático. 
Palabras clave: App, discapacidad visual, energía solar, módulos inalámbricos wifi, terminal móvil.

\begin{abstract}
The present work describes the Design and elaboration of a prototype that by means of an algorithm links with a mobile application (App) and allows the orientation of visually impaired people implemented in the Faculty of Computer Science of the Technical University of Manabí; it consists of two parts, the mobile application which was developed in the programming environment Android Studio that allows detecting wireless signals (standard IEEE 802.11); the device detects certain pre-programmed signals at a certain distance will emit an audio that will be accompanied by a different vibration for each reference point indicating the sector in which it is circulating. The other part of the prototype are the reference points or access points, whose main component is a Wireless module (NodeMcu V3 LoLin), which through an algorithm emits signaling in the ISM band of $2.4 \mathrm{GHZ}$ to mobile terminals. The reference points have energy autonomy of up to 20 hours from the alternating current supply by means of a photovoltaic panel in an automatic switching circuit.
\end{abstract} Keywords: App, mobile terminal, solar energy, Visual impairment, WiFi wireless modules.

\section{Resumo}

O presente trabalho descreve a concepção e elaboração de um protótipo que, por meio de um algoritmo de ligações com uma aplicação móvel (App) e permite a orientação de pessoas com deficiência visual implementado na Faculdade de Ciências da Computação da Universidade Técnica de Manabí; consiste em duas partes, a aplicação móvel que foi desenvolvido no ambiente de programação Android Studio que permite detectar sinais sem fio (padrão IEEE 802.11); o dispositivo detecta certos sinais pré-programados a uma determinada distância e emitirá um áudio que será acompanhado de uma vibração diferente para cada ponto de referência, indicando o sector em que está a circular. A outra parte do protótipo são os pontos de referência ou pontos de acesso, cujo componente principal é um módulo Wireless (NodeMcu V3 LoLin), que a través de um algoritmo emite sinalização na banda ISM de $2.4 \mathrm{GHZ}$ para terminais móveis. Os pontos de referência têm uma autonomia energética de até 20 horas em relação à alimentação de corrente alterna a través de um painel fotovoltaico num circuito de comutação automática.

Palavras-chaves: App, Deficiência visual, energia solar, módulos WiFi sem fios, terminal móvel.

\section{Introducción}

En el mundo el desarrollo constante de la tecnología ha sido producto de los nuevos equipos y plataformas que se actualizan continuamente. Esto permite a los investigadores desarrollar proyectos que permitan satisfacer necesidades ya sea en el ámbito técnico, académico o social. Al ritmo que evolucionan las actividades tecnológicas se pretende desarrollar nuevas herramientas para ser introducidas en una sociedad incluyente: tecnologías al alcance de todos que mejoren la calidad de vida de las personas (Acosta, Lozano y Rico, 2015).

La tecnología asistencial para las personas ciegas y con discapacidad visual es un campo de investigación que está ganando cada vez más protagonismo debido al elevado interés por parte de distintas disciplinas convergentes; ello acompañado de un impacto social muy pertinente en nuestra población. Concebir las necesidades de una persona con discapacidad visual puede resultar en un análisis muy complejo pues, dependiendo de las capacidades de los sentidos que implique esta, podrá haber otras discapacidades de los sentidos que pueden dificultar la movilidad del individuo.

La cifra estimada de personas con discapacidad visual es de 253 millones: 36 millones con ceguera y 217 millones con discapacidad visual moderada a grave (Bhoir, Abraham y Wadhaiya, 2016). Además, uno de los grandes desafíos para los usuarios con discapacidad visual es el tránsito por las zonas urbanas exteriores, especialmente si no está familiarizada con la locación. Se observa que hay una serie de zonas difíciles y potencialmente peligrosas para que una persona pueda transitar como, por ejemplo, cruzar una vía transitable. 
Para detectar obstáculos, los sistemas de asistencia sensorial utilizan métodos de navegación local utilizados en el campo de la robótica, así como métodos de tránsito de personas en general basados en la supervisión y la comunicación de información a distancia (Kulyukin y Kutiyanawala, 2010). El proceso de guiado difiere sustancialmente en el caso del entorno exterior con respecto al ambiente interior. Algunos sistemas proporcionan orientación para desplazarse con el transporte público (Dong et al., 2014), mientras que otros se centran en guiar en entornos interiores desconocidos (Tapu et al., 2013).

El surgimiento de dispositivos móviles en los últimos años, aunado con el desarrollo de la tecnología, ha hecho posible la aparición de sistemas de asistencia basados en algoritmos que identifican objetos relevantes como el dinero o para ayudar en las compras de supermercado (Korial y AbduIlah, 2016; Mugyenyi, Evarist y Bamutura, 2016). Para una buena orientación en entornos interiores, se han utilizado dispositivos con RFID (Ashraf y Raza, 2014; Cruz et al., 2018) y también la cámara del móvil para detectar obstáculos mediante un sistema de clasificación de objetos (Pampattiwar y Chhangani 2014).

Por tal motivo, como parte los requerimientos de este proyecto, se realizó la investigación aplicando técnicas de observación y entrevistas a personas con discapacidad visual de la Universidad Técnica de Manabí, en la cual se percibió la necesidad de facilitarles una orientación asistida por medio del dispositivo móvil y puntos de red como referencia del lugar en el que van desplazándose, para que tengan una mayor autonomía de desplazamiento en la zona circulante y así llegar a su destino con facilidad.

\section{Metodología}

Varios trabajos de investigación han realizado diferentes aplicaciones de software en el dispositivo móvil. Algunos de ellos utilizando solo la parte de hardware del teléfono, otros utilizando también módulos de hardware externos (Méndez y Cuervo, 2011). Sin embargo, crear una App para personas con discapacidad visual implica crear una interfaz diferente a la habitual (Cruz et al., 2018; Fernandez, 2010).

Para desarrollar la estructura de los sensores se necesitó del análisis de dispositivos, lugar, ambiente y otros factores que influyen en el proyecto. Como primera parte se seleccionó los sensores, estos son módulos wifi.

\section{Módulo ESP 8266 (NodeMcu V3 LoLin)}

El módulo wifi escogido es desarrollado por Aithinker Team (Shenzhen Anxinke Technology LTD CO., 2015) cuenta con un Procesador central ESP8266, integra un micro MCU de ultra bajo consumo de 32 bits. Trabaja según el estándar IEEE802.11 b / g / n, siendo compatible con la pila de la arquitectura TCP / IP. Los usuarios pueden usar los módulos para agregar a una red de dispositivos existentes o construir un controlador de red separado. El módulo ESP8266 es SOC inalámbrico de alta integración, concebido para diseñadores de plataformas móviles con limitaciones de espacio y energía (Bourne et al., 2017).

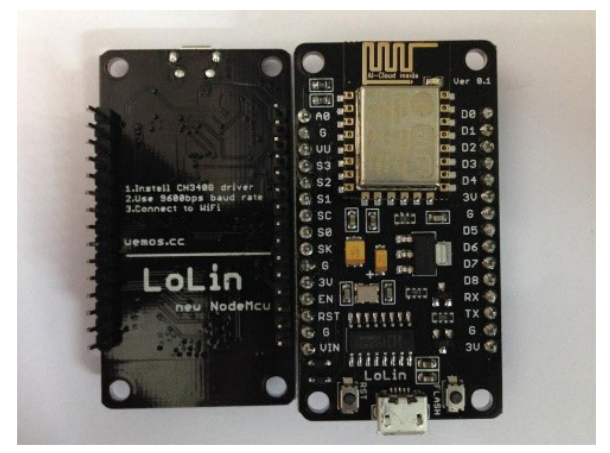

Figura 1. Módulo wifi ESP8266 V3.

Fuente: elaboración propia del autor.

Se escogió este módulo (figura 1) por sus características óptimas para el objetivo del proyecto. Además, es pequeño y útil por su alimentación de $5 \mathrm{~V}$ y tiene bajo consumo de energía: 
- Consumo de corriente en modo de espera @ $80 \mathrm{MHz}: 80 \mathrm{~mA}$.

- Consumo de corriente al recibir una petición (librería WebServer en modo de punto de acceso) @80MHz: $90 \mathrm{~mA}$.

- Consumo de corriente al utilizar HTTPClient. get() @80MHz: 100-110 mA.

- Consumo de corriente en modo de espera @160MHz: 90 mA.

- Consumo de corriente al recibir una petición (librería WebServer en modo de punto de acceso)@ 160MHz: 90-100 mA.

- Consumo de corriente al utilizar HTTPClient. get() @ 160 MHz: 100-110 mA para su funcionamiento idóneo.

\section{Energía solar fotovoltaica-El panel solar}

Otro de los requisitos que se analizó fue agregar un sistema de alimentación de energía al dispositivo desde una batería que funcione con carga de energía renovable. La energía solar fotovoltaica es una fuente de energía renovable, se la considera inagotable, limpia y se puede aprovechar en el mismo lugar en que se produce (auto gestionada) (Helal, Moore y Ramachandra, 2001). Una sola célula no es capaz de proporcionar una tensión que pueda utilizarse en la práctica, solo genera una tensión de algunas décimas de voltio (usualmente, alrededor de medio voltio para las células de silicio), y una potencia máxima de uno o dos vatios. Para obtener tensiones y potencias adecuadas es preciso conectar entre sí en serie un determinado número de células para producir tensiones de 6, 12 o 24 voltios, aceptadas en la mayor parte de las aplicaciones. A este conjunto de células interconectadas, ensambladas y protegidas contra los agentes externos, se le denomina panel o módulo fotovoltaico.

Según el análisis realizado, se determinó usar un panel solar poli cristalino (figura 2) con las siguientes características:

- Número de celdas: 1

- Material: silicona policristalina
- Capacidad nominal: 9V/ $2 \mathrm{~W}$

- Tamaño: 11.5 x $11.5 \mathrm{~cm}$

- Máxima potencia: $2 \mathrm{~W}$

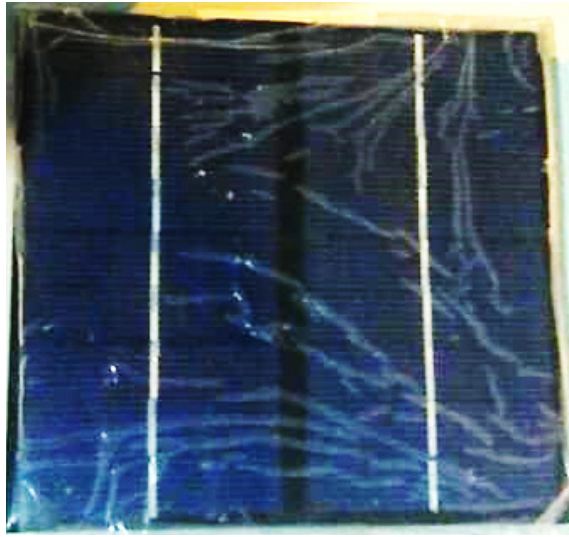

Figura 2. Panel solar de $9 \mathrm{~V} 220 \mathrm{mAh}$.

Fuente: elaboración propia del autor.

La batería es otro de los elementos principales para el funcionamiento de los módulos inalámbricos wifi, para ello se utilizó la batería de polímero de litio de $2500 \mathrm{~mA}$, con una tensión nominal de $3.8 \mathrm{~V}$ (figura 3 ).

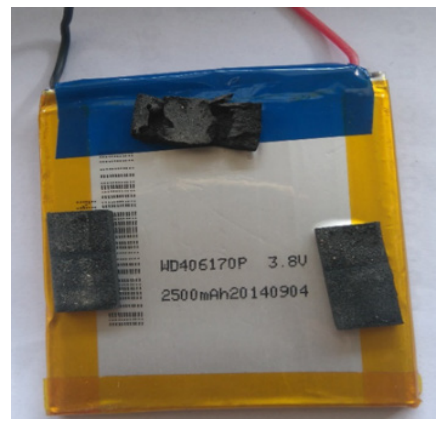

Figura 3. Batería.

Fuente: elaboración propia del autor.

\section{Diseño del circuito para el funcionamiento del módulo con corriente alterna o corriente conti- nua alimentada por panel solar}

Para alimentar al módulo ESP 8266 wifi se diseñó un circuito que permite el funcionamiento del módulo el mayor tiempo posible. Realizando un circuito conectado a la corriente alterna, el cual se compone de un transformador de 110v a $6 \mathrm{v}$ en 
AC, un fusible de protección, un puente de diodo para convertir la energía alterna en energía directa, condensadores para eliminar los picos de voltaje y un transistor regulador KIA7805 para entregar el voltaje optimo al módulo wifi, también se incorporó un circuito de conmutación, el cual (al detectar la ausencia de energía alterna) realiza el cambio de fuente y empieza a trabajar con la batería de respaldo la cual es cargada con energía solar.

El circuito de regulación de tensión para alimentar al módulo de carga de batería a través del panel solar cuenta con diodos de protección 1n4148, el cual soporta un voltaje máximo inverso de $100 \mathrm{~V}$ y corriente directa de 500 mA (Monther et al., 2014). También, se tiene dos condensadores de $6800 u F / 10 \mathrm{~V}$ en la entrada para mejorar su respuesta al transistor regulador de tensión KIA7805, el cual envía la tensión necesaria al módulo de carga de la batería (figura 4).

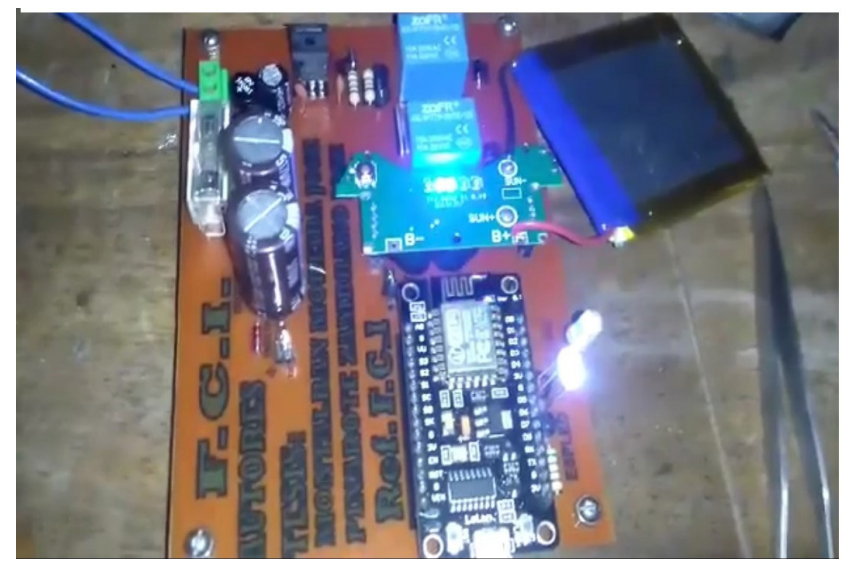

Figura 4. Circuito de conmutación de alimentación eléctrica.

Fuente: elaboración propia del autor.

\begin{tabular}{|c|c|c|c|c|}
\hline 1 & Hora & Nivel Bateria & Intensidad Sol & Led \\
\hline 2 & 09928 & 3,816 & 2 & 4 \\
\hline 3 & $10 \mathrm{~h} 06$ & 3,802 & 3 & 4 \\
\hline 4 & $10 \mathrm{~h} 37$ & 3,788 & 2 & 4 \\
\hline 5 & $10 \mathrm{~h} 38$ & 3,797 & 2 & 4 \\
\hline 6 & 1 tho0 & 3,784 & 2 & 4 \\
\hline 7 & 1 th 20 & 3,757 & 2 & 4 \\
\hline 8 & 1hh37 & 3,767 & 2 & 4 \\
\hline 9 & $12 \mathrm{~h} 01$ & 3,758 & 2 & 4 \\
\hline n & $02 \mathrm{~h} 06$ & 3,688 & 1 & 4 \\
\hline 11 & $02 \mathrm{~h} 47$ & 3,66 & 1 & 4 \\
\hline 12 & $03 \mathrm{~h} 08$ & 3,649 & 1 & 4 \\
\hline 13 & $04 h 55$ & 3,571 & 1 & 4 \\
\hline 14 & 0Sh11 & 3,562 & 1 & 3 \\
\hline 15 & $06 \mathrm{~h} 05$ & 3,542 & 0 & 3 \\
\hline 18 & $07 \mathrm{~h} 03$ & 3,519 & 0 & 3 \\
\hline 17 & $07 h 40$ & 3,5 & 0 & 3 \\
\hline 18 & $08 \mathrm{~h} 26$ & 3,483 & 0 & 3 \\
\hline 19 & $08 \mathrm{~h} 47$ & 3,482 & 0 & 3 \\
\hline 20 & $1 \mathrm{~h} 33$ & 3,433 & 0 & 3 \\
\hline 21 & 1hh58 & 3,427 & 0 & 2 \\
\hline 22 & $12 h 53$ & 3,406 & 0 & 2 \\
\hline 23 & Ozhil & 3,372 & 0 & 2 \\
\hline 24 & O2h34 & 3,363 & 0 & 2 \\
\hline 25 & $02 h 50$ & 3,354 & 0 & 2 \\
\hline 28 & $03 \mathrm{~h} 30$ & 3,249 & 0 & 2 \\
\hline 27 & $04 h 00$ & 3,225 & 0 & 2 \\
\hline 28 & $05 \mathrm{~h} 40$ & 3,142 & 0 & 1 \\
\hline 29 & $06 \mathrm{~h} 10$ & 3,044 & 0 & 1 \\
\hline 30 & $07 h 06$ & 2,894 & 0 & 1 \\
\hline 31 & $07 \mathrm{~h} 30$ & 2,786 & 0 & 1 \\
\hline 32 & $07 \mathrm{~h} 38$ & 2,783 & 0 & 0 \\
\hline
\end{tabular}

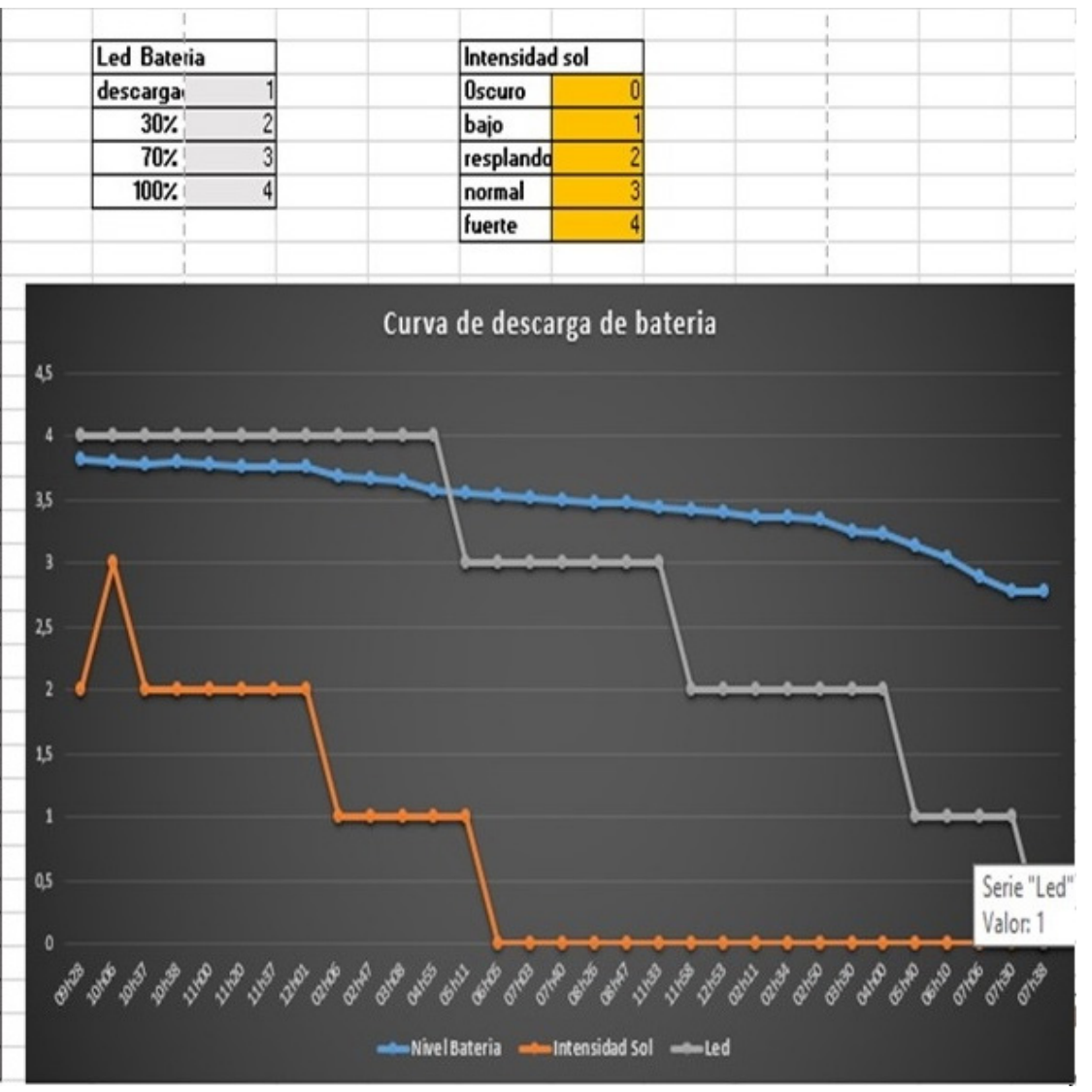

Figura 5. Curva de valores de descarga de la batería con panel solar.

Fuente: elaboración propia del autor. 
La batería de polímero de litio tiene un tiempo de duración de 24 horas, que dependerá de la intensidad de los rayos de sol en la cual se realice el proceso de descarga por el uso de la misma y tendrá un periodo de carga de 6 a 12, horas dependiendo de la intensidad de los rayos de sol.

En la figura 5 se observa la curva de carga y en la figura 6 se muestra la curva de descarga de acuerdo con los valores tomados en el desarrollo de proyecto. La curva de color naranja representa la intensidad del sol proporcional al voltaje que genera y la curva color azul representa el estado de carga de la batería; estos datos fueron tomados en el caso extremo de un día nublado con poca radiación solar.

Se realizaron las mediciones con el objetivo de comprobar las características de la batería, pensando en que los nodos o puntos de referencia inalámbricos wifi estén siempre encendidos.

\section{Estructura de los nodos inalámbricos wifi como punto de referencia}

Los módulos wifi ESP8266 (NodeMcu V3 LoLin) actuan como puntos de referencia colocados en lugares estratégicos escogidos previamente para poner en funcionamiento el prototipo del proyecto. Estos dispositivos van a mantener una señal de radio constante en el tiempo, pero variable en su intensidad dependiendo de las condiciones del ambiente. La potencia de la señal en decibeles es captada por la aplicación móvil que estará de manera constante buscando las señales inalámbricas.

En la figura 7 se encuentran tres nodos que están enviando señal inalámbrica en la frecuencia de 2.4 GHZ y la representación de la persona que está circulando por esa zona con su dispositivo móvil escaneando las redes vinculadas a la aplicación. Dicha aplicación tiene un algoritmo para

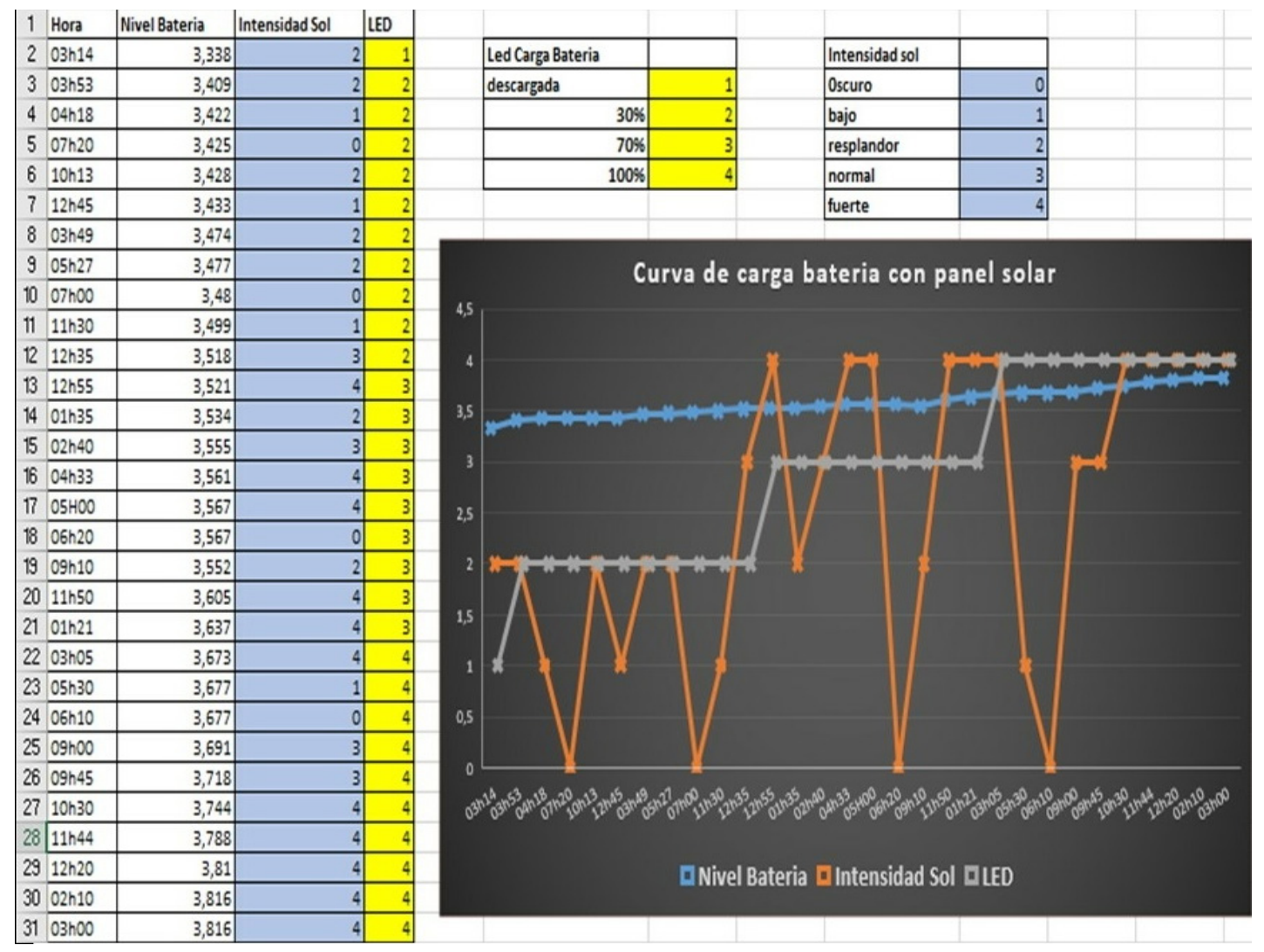

Figura 6. Curva de valores de carga de la batería con panel solar.

Fuente: elaboración propia del autor. 
interpretar las señales de los nodos instalados en el área, con lo cual se envían señales sonoras y de vibración que alertan a la persona con discapacidad visual un lugar de referencia donde se encuentre.
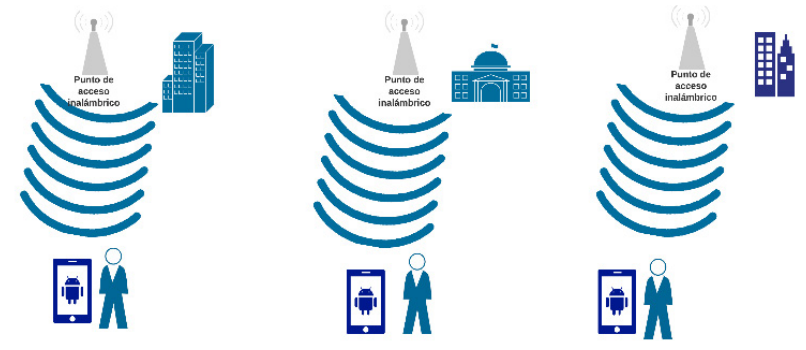

Figura 7. Nodos inalámbricos wifi como puntos de referencia.

Fuente: elaboración propia del autor.

La aplicación móvil App está diseñada para detectar la señal inalámbrica wifi y en las pruebas realizadas se optimizó el algoritmo para que envié la señal audible en niveles de potencia al usuario. Esto permitirá que el dispositivo móvil que detecta la señalización se active cuando el nivel de la ganancia de la señal sea igual a la establecida para el modelo del móvil en uso. Por ejemplo, en el caso particular del móvil marca Sony modelo Xperia Z5 se estableció el nivel de señal en $-65 \mathrm{dBm}$, siendo la ideal para que el usuario reciba la señal en un rango de entre 4 a 6 metros antes de pasar el módulo inalámbrico wifi y de entre 4 a 6 metros después de haber pasado por el mismo.

\section{Aplicación móvil}

La aplicación móvil que se ha creado tiene como nombre Clover y ha sido especialmente diseñada para este tipo de usuarios; para ser útil en la asistencia a la persona con discapacidad visual. No se recomienda utilizar el altavoz para no molestar a las personas que lo rodean, y el auricular debe ser usado solo para un oído para que pueda oír con el otro los ruidos ambientales y del entorno que puede ser útiles.

La aplicación se desarrolló en el entorno de programación Android Studio para ser instalada en un dispositivo móvil con sistema operativo Android con una versión 4.0 o superior. Esta aplicación está diseñada para realizar búsquedas consecutivas de los puntos de referencia inalámbricos wifi; una vez encontrados dichos puntos y estar cerca del rango al cual fue programada la sensibilidad activara un audio que a su vez estará seguida de vibraciones de manera distinta para cada nodo o punto de referencia y de esta manera las personas con discapacidad visual puedan asimilar que cada audio y vibración pertenece a un sector distinto por el cual se encuentra transitando.

Para instalar la aplicación en el dispositivo móvil se debe habilitar el modo desarrollador de la siguiente manera:

1. Ingrese a Menú.

2. Ingrese a Configuración o Ajustes.

3. Ingrese "Acerca" del teléfono o dispositivo.

4. Presione Número de compilación 7 veces.

5. Presione una vez regresar.

6. Ingrese a Opciones de desarrollador.

7. Active modo de depuración USB.

Luego se conecta el USB al terminal del PC en la cual se encuentra la aplicación desarrollada. En el dispositivo móvil aparecerá un mensaje que pide “¿Permitir depuración por USB?", se tendrá que aceptar por lo tanto la huella digital de la clave RSA y se hará clic en "permitir siempre desde este ordenador" (figura 8).

Este proceso se tendrá que realizar en cada dispositivo móvil que no tenga habilitada la huella permitida, por seguridad. En Android Studio, se debe habilitar Google USB driver para poder instalar el controlador adecuado para el dispositivo móvil a utilizar y así lo reconozca y pueda desplegar la App en el dispositivo y luego depurarla de la siguiente manera:

1. File

2. Settings

3. Apperance \& Behavior

4. System Settings

5. Android SDK 
6. Seleccionar la pestaña SDK Tools

7. Marcar la opción Google USB Driver Clic en Apply

Ahora, reconocerá el dispositivo móvil en Android Studio y al seleccionar el dispositivo y hacer clic en "Ok" la App se lanzará en nuestro móvil (figura 9).

\section{Resultados}

Las pruebas funcionales se realizaron de manera progresiva, cada vez que se tenía una mejora se realizaban nuevas pruebas en el área real en la que se ubicarían los puntos de referencia para lograr obtener una aplicación 100 \% funcional.

Para constatar la funcionalidad de la aplicación se escogieron aleatoriamente varias personas con discapacidad visual, en su mayoría estudiantes de la Universidad Técnica de Manabí. Dichas personas hicieron el recorrido por los módulos inalámbricos instalados, como se aprecia en la figura 10.

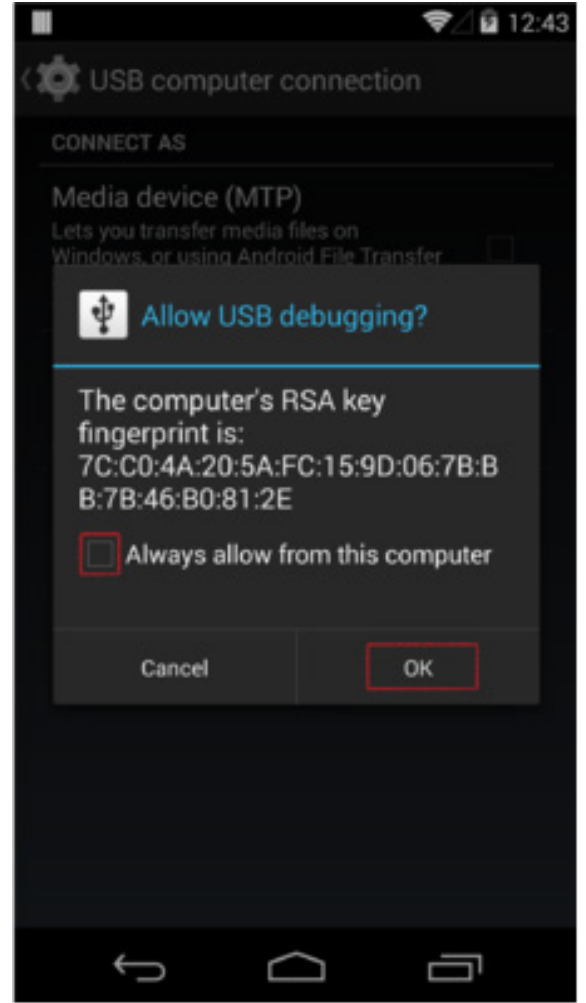

Figura 8. Permitir depuración por USB. Fuente: elaboración propia del autor.

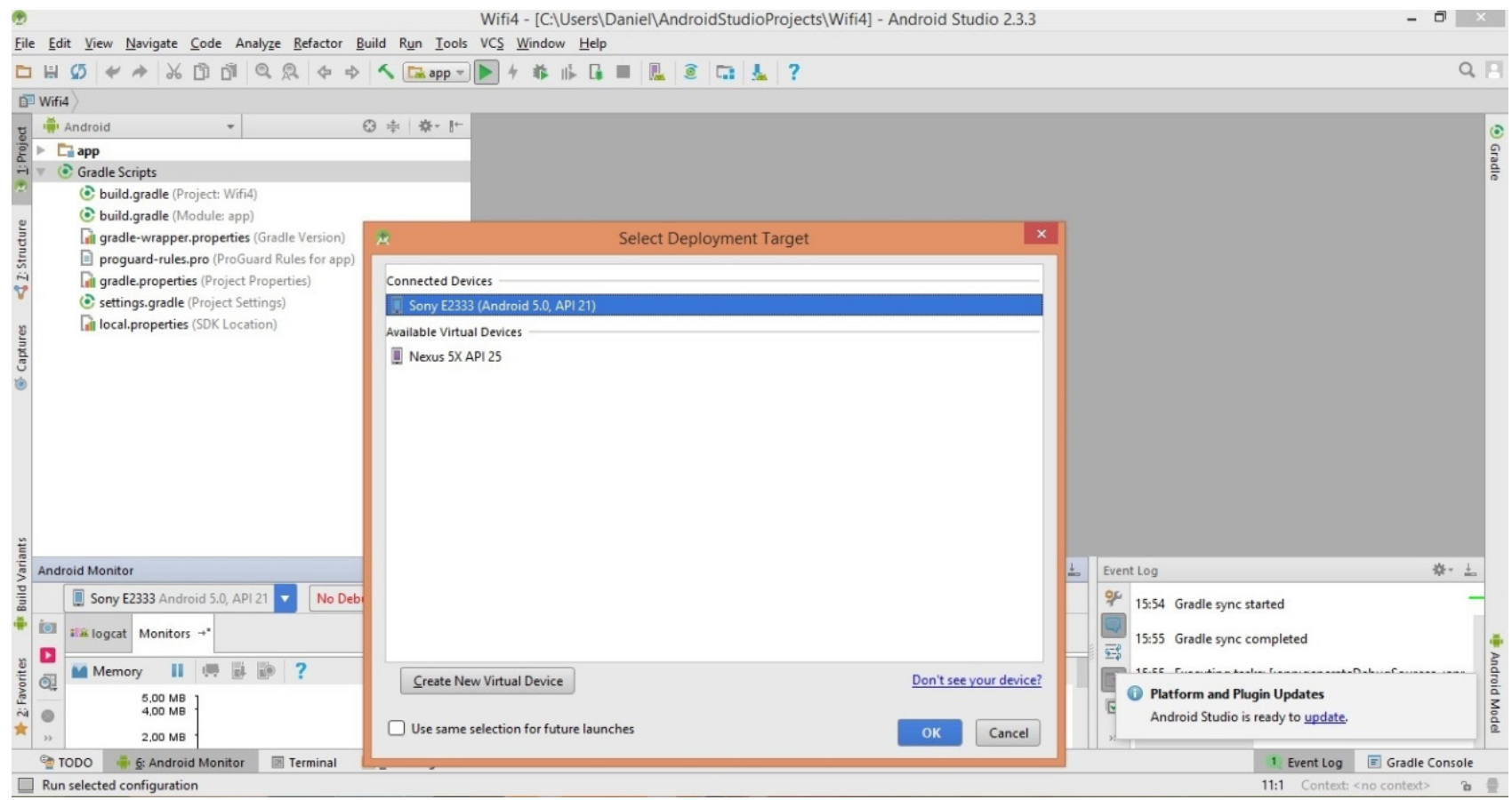

Figura 9. Reconocimiento del móvil en Android Studio.

Fuente: elaboración propia del autor. 
Las pruebas del prototipo y la aplicación Android se realizó utilizando varios modelos de dispositivos móviles. Después de las pruebas, se determinó el rendimiento del sistema y se obtuvo que la interfaz de aplicación era fácil de usar, la información comunicada era bien comprendida incluso cuando el ruido ambiental era más alto, en especial porque la persona con discapacidad visual tiene una capacidad auditiva más desarrollada. Como el trayecto se da en el entorno exterior, se requería ocasionalmente una reducción de la velocidad cuando, en algunas áreas, la señal del teléfono era débil. De manera general, el sistema ha demostrado su eficacia en tiempo real.

\section{Conclusiones}

Para ayudar a las personas con discapacidad visual los dispositivos móviles resultan ser muy útiles, pero se requiere módulos sensoriales externos al teléfono para detectar obstáculos y encontrar una manera segura de transitar.

La aplicación en Android diseñada ofrece orientación en el trayecto y la interfaz especial creada para los personas con discapacidad visual ha demostrado su eficacia. Por esto, la comunicación de la información pertinente transmitida mediante los sonidos a un oído conduce a una correcta comprensión del mensaje y otorga a las personas con discapacidad visual la oportunidad de recibir otra información útil del entorno.

Los nodos inalámbricos transmiten los datos al terminal móvil mediante wifi, realizando una fusión de datos sensoriales, y en el momento oportuno se comunica al usuario no vidente la información relevante, avisando de su ubicación con respecto a un punto de referencia.

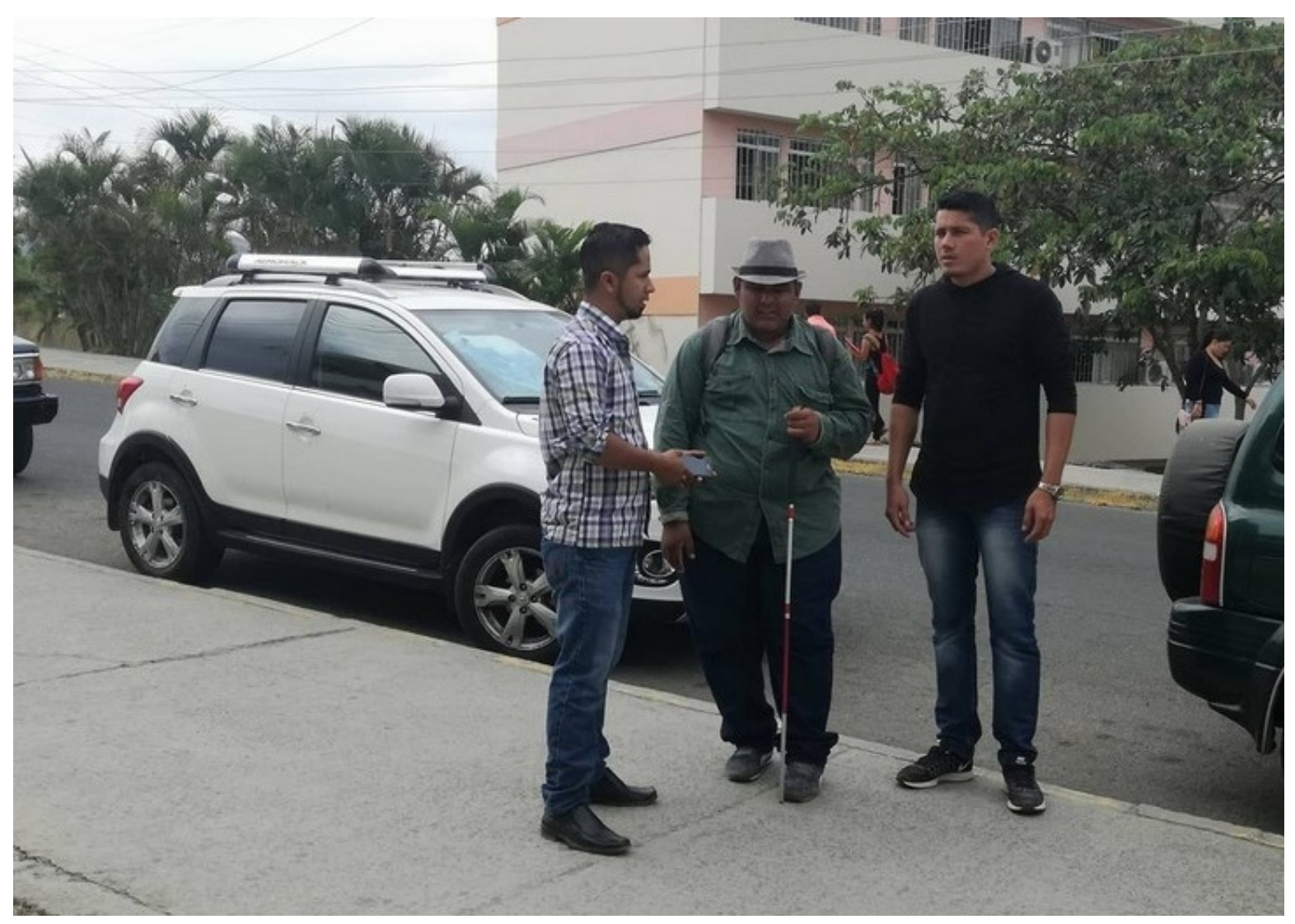

Figura 10. Prueba del prototipo con una persona no vidente.

Fuente: elaboración propia del autor. 
Se demostró que se puede brindar ayuda a personas con discapacidad visual usando un dispositivo móvil y módulos wifi como puntos de referencia y con esto plasmar que es factible usar tecnología de bajo costo y fácil uso, debido a que la compatibilidad de los módulos con la aplicación resultó ser muy efectiva, ya que con muy poco entrenamiento se logró brindar ayuda a los usuarios no videntes.

Las pruebas experimentales realizadas con el prototipo han demostrado su utilidad, pero también han revelado que se necesitan más pruebas para encontrar la distancia óptima de detección de la ubicación en los entornos cercanos a los edificios como en el ambiente exterior en vías transitadas.

En un futuro se tendrá dispositivos móviles con más capacidad de procesamiento y potencia los cuales, además de abaratar costos, se viabilizará una asistencia más óptima y eficaz para las personas con discapacidad visual.

\section{Referencias}

Acosta-López, A., Lozano, H. y Rico, C. (2015). Diseño e implementación de un prototipo de sistema de identificación por radiofrecuencia para la verificación de información de vehículos. Redes de Ingeniería, 6(2), 3343. $\quad$ https://doi.org/10.14483/udistrital.jour. redes.2015.2.a04

Al-Shehabi, M., Mustahsan, M., Abdullah, M. y Ahmed, M. (2014). An Obstacle Detection and Guidance System for Mobility of VisuaIly Impaired in Unfamiliar. International Journal of Computer and Electrical Engineering, 337- 341. https://doi.org/10.7763/IJCEE.2014. V6.849

Ashraf, A. y Arif, R. (2014). Usability Issues of Smart Phone Applications: For Visually ChaIlenged People. World Academy of Science, Engineering and Technology, 760-767.

Bhoir, S., Ajeesh, A. y Krupa, W. (2016). Camera based product identification for the visually impaired. International Journal of Engineering Research and General Science, 413-417.

Bourne, R., Flaxman, S., Braithwaite, T., Cicinelli, M. V., Das, A. y Jonas, J. B. (2017). Vision Loss Expert Group. Magnitude, temporal trends, and projections of the global prevalence of blindness and distance and near vision impairment: a systematic review and meta-analysis. The Lancet Glob Health, 888-897.

Cruz, F, Pinargote Ortega, M., Demera, G., Vera-Zambrano, E. y Mosquera-Alcivar, R. (2018). Sistema de alerta para estudiantes con discapacidad visual en la UTM. Revista Científica, 31(1), 85-95. https://doi. org/10.14483/23448350.12464

Dong, H., Kang, J., Schafer, J. y Ganz, A. (2014). Android-Based Visual Tag Detection for VisuaIly Impaired Users: System Design and Testing. International Journal of EHealth and Medical Communications, 5(1), 63-80.

Fernandez, M. (2010). Energía solar: electricidad fotovoltaica. Madrid: Liber Factory.

Helal, A, Moore, S. E. y Ramachandra, B. (2001). Drishti: an integrated navigation system for visually impaired and disabled. Proceedings Fifth International Symposium on Wearable Computers, 149-156. https://doi.org/10.1109/ ISWC.2001.962119

Korbel, P., Skulimowski, P., Wasilewski, P. y Wawrzyniak, P. (2013). Mobile applications aiding the visually impaired in travelling with public transport. Federated Conference on Computer Science and Information Systems (pp. 825-828).

Korial, A. y Mohammed A. (2016). Novel Method using Beacon and Smart Phone for Visually Impaired/Blind People. International Journal of Computer Applications, 137(1), 33-39. https:// doi.org/10.5120/ijca2016908674

Kulyukin, V. y Aliasgar K. (2010). Accessible Shopping Systems for Blind and Visually Impaired Individuals: Design Requirements and the State of the Art. The Open Rehabilitation Journal, 
3(1), 158-168. https://doi.org/10.2174/187494 $\underline{3701003010158}$

Méndez, J. y Cuervo, R. (2011). Energía solar fotovoltaica. Madrid: FC Editorial. Fundación Confemetal.

Mugyenyi, R., Nabaasa, E. y Bamutura, D. (2016). Enhanced Protocols for Non-Visual Navigation of Smartphone Applications Screen Icons. International Journal of Computer Trends and Technology, 38(3), 119-123. https://doi. org/10.14445/22312803/IJCTT-V38P121

Pampattiwar, R. y Anil Chhangani. (2014). Smartphone Accessibility Application for Visually
Impaired. International Journal of Research in Advent Technology, 2(4), 377-380.

Shenzhen Anxinke Technology LTD, CO. (2015). ESP-12E WiFi Module, versión 1.0. http:// www.kloppenborg.net/images/blog/esp8266/ esp8266-esp12e-specs.pdf

Tapu, R., Mocanu, B., Bursuc, A. y Zaharia, T. (2013). A Smartphone-Based Obstacle Detection and Classification System for Assisting Visually Impaired People. Proceedings of the IEEE International Conference on Computer Vision Workshops, 444-451. https://doi.org/10.1109/ ICCVW.2013.65 